\title{
Urgencias en ginecología oncológica
}

\section{Emergencies in oncological gynaecology}

\author{
J.C. Muruzábal, S. Aguirre, S. Aranda, F.J. Elizalde
}

\section{RESUMEN}

En este trabajo se describen los signos de sospecha de neoplasia por los que la paciente acude al Servicio de Urgencias, centrándonos en el sangrado genital, la masa pélvica y el prurito vulvar. Los pacientes también pueden acudir por procesos resultantes de complicaciones de la enfermedad, como en el caso de la obstrucción ureteral, la carcinomatosis, la ascitis, procesos tromboembólicos, hemorragias, estreñimiento, náuseas y vómitos, obstrucción intestinal y dolor. Por último, se describen complicaciones secundarias al tratamiento como el linfocele abdominal e inguinal y la enteritis y proctitis postradioterápica.

Palabras clave. Complicaciones oncológicas. Urgencias ginecológicas. Enfermedad tromboembólica. Masa anexial. Metrorragia postmenopáusica.

\begin{abstract}
This article describes the signs for suspecting neoplasia that lead the patient to come to the Accident and Emergency Service, concentrating on genital bleeding, pelvic mass and vulvar pruritus. Patients can also come due to processes resulting from complications of the disease, such as urethral obstruction, carcinomatosis, ascites, thromboembolic processes, haemorrhages, constipation, nausea and vomiting, intestinal obstruction and pain. Finally, we describe complications that are secondary to the treatment, such as abdominal and inguinal lymphocele and post-radiotherapy enteritis and proctitis.
\end{abstract}

Key words. Oncological complications. Oncological emergencies. Thromboembolic disease. Adnexal mass. Postmenopausal bleeding.
Servicio de Obstetricia y Ginecología. Hospital Virgen del Camino. Pamplona.

\section{Correspondencia:}

Juan Carlos Muruzábal Torquemada

Sancho el Fuerte $49,5^{\circ} \mathrm{D}$

Tfno. 607512929

31008 Pamplona

E-mail: jcmuruzabal@hotmail.com 


\section{INTRODUCCIÓN}

Las urgencias oncológicas se definen como aquellas situaciones que, implicando un riesgo para la vida del paciente o causando un deterioro agudo de su estado de salud, están directa o indirectamente relacionadas con la enfermedad neoplásica o su tratamiento.

En el manejo de una situación urgente en un paciente oncológico deben de hacerse una serie de consideraciones, a saber: la reversibilidad del proceso y la posibilidad de curación, la expectativa de vida del paciente y la disponibilidad de un tratamiento paliativo, valorando la relación riesgo/ beneficio que su aplicación conlleva.

En el período 1998-2002, se registraron 16.952 casos de cáncer en Navarra, de los que 9.864 (58\%) fueron diagnosticados en hombres y 7.088 (42\%) en mujeres. En las mujeres, el cáncer de mama es el tumor maligno más frecuentemente diagnosticado $(28,1 \%)$, seguido por el cáncer colorrectal $(13,9 \%)$, cáncer de cuerpo de útero $(6,7 \%)$, estómago $(4,7 \%)$ y ovario $(4,2 \%)$. Además de dichos tumores malignos, se contabilizaron 1.334 tumores "in situ" o de malignidad incierta en las siguientes localizaciones: 421 casos en vejiga, 358 en piel, 229 en cérvix uterino, 194 en mama y el resto en otras localizaciones ${ }^{1}$.

Otra de las informaciones que nos ayudan a aproximarnos a la magnitud del problema en España es el de la repercusión asistencial del paciente oncológico. Los datos actuales de la Encuesta de Morbilidad Hospitalaria muestran un $10 \%$ de ocupación de plaza hospitalarias por pacientes con tumores malignos, elevándose a un $14 \%$ en el grupo de edad entre 45 y 64 años ${ }^{2}$.

\section{SOSPECHA DE NEOPLASIA EN URGENCIAS}

\section{Sangrado genital}

Se puede observar sangrado genital en metrorragia postmenopáusica (sangrado uterino que aparece tras 12 meses de amenorrea, con niveles elevados de gonadotropinas), mujeres que presentan un sangrado durante el tratamiento con tamoxifeno por cáncer de mama, así como aquellas que re- ciben tratamiento hormonal sustitutivo y presentan un cambio en el patrón de sangrado.

En mujeres menopáusicas normalmente tiene un origen uterino, pero se deben descartar otros orígenes:

- Origen urológico: infección urinaria, prolapso mucosa uretral. Realizar sondaje vesical y exploración urológica.

- Origen digestivo: lesiones anales y rectales. Realizar tacto rectal.

- Origen ginecológico: vulvovaginitis atrófica, lesiones vulvares sugestivas de neoformación, traumatismos, pólipos cervicales, cáncer clínico de cérvix uterino, hemorragia uterina anómala.

Es un motivo frecuente de consulta y se debe descartar patología maligna ya que es el síntoma más frecuente del adenocarcinoma de endometrio ${ }^{3}$ y siempre debe ser realizado un estudio completo siguiendo los siguientes pasos:

- Anamnesis: detallada, que nos orientará hacia el diagnóstico.

- Exploración física: se valorará la repercusión clínica (TA, frecuencia cardíaca, $\mathrm{T}^{\mathrm{a}}$, coloración, nivel de conciencia...). Se realizará una exploración general, una exploración abdominal y una exploración ginecológica dirigida a localizar el origen del sangrado con inspección de genitales externos, vulva y periné, espéculo para el diagnóstico de posible patología vaginal y cervical y tacto bimanual.

- Analítica: hemograma completo con plaquetas en toda paciente ${ }^{4}$. El valor de la $\mathrm{Hb}$ y Hto. determinará la severidad del proceso; también valoración de la hemostasia.

- Estudio ecográfico: técnica de gran utilidad en el diagnóstico ginecológico. Es la prueba más eficiente por ser bien tolerada, no invasiva y, por lo tanto, sin complicaciones, fácilmente accesible para cualquier especialista y que permite la detección de patología uterina y ovárica. El espesor normal del endometrio está en función de la situación pre o posmenopáusica, fase del ciclo y toma de fármacos como THS y tamoxifeno. La medición del grosor en- 
dometrial es la variable más usada, ya que en muchos estudios se ha comprobado que según el punto de corte su sensibilidad para detección del cáncer de endometrio varía entre 68 y $100 \%$. Ya en el estudio realizado por Karlsson en 1995 se estableció el riesgo de encontrar patología endometrial cuando el grosor del endometrio es menor o igual a $4 \mathrm{~mm}$ es de $5 \%$. Mossa determina que en pacientes con THS se establece el punto de corte en $8 \mathrm{~mm}$ para pacientes con metrorragia, por encima del cual se debe realizar estudio anatomopatológico ${ }^{6}$.

- Estudio histológico: se debe realizar biopsia de las lesiones sospechosas que se encuentran durante la exploración de vulva, vagina y cérvix. No se recomienda estudio de forma generalizada en la evaluación inicial del sangrado uterino anómalo. Indicado en: mujer mayor de 35 años, hemorragia persistente, fracasos de tratamiento, factores de riesgo para el cáncer de endometrio y antes de un tratamiento quirúrgico. Opciones :

1. Biopsia endometrial: mediante microlegrado aspirativo (la técnica más utilizada es la cánula de Cournier, microlegra) puede ser diagnóstica si el material es suficiente, pero no proporciona información sobre la cavidad endometrial y por ello puede tener falsos negativos. Presenta escasas complicaciones, se puede realizar en la misma consulta y es bien tolerada.

2. Biopsia dirigida mediante histeroscopia diagnóstica: es el siguiente paso, y se debe remitir a las pacientes desde urgencias a la consulta. Es la técnica más precisa.

\section{Tratamiento}

Siempre que sea posible, el tratamiento será etiológico y generalmente podrá ser realizado de forma ambulatoria, pero dependiendo de la repercusión general podrá ser necesaria la asistencia en el servicio de urgencias o ingreso hospitalario.

Tratamiento ambulatorio: tratamiento médico a corto-medio plazo, hormonal-no hormonal para la hemorragia y se remitirá a la consulta. No olvidar el tratamiento de la anemia

Paciente que precisa ingreso: estabilizar hemodinámicamente a la paciente, monitorización, aporte de volumen y transfusión si precisa. Simultáneamente a este tratamiento general, se procederá a tratamiento médico o quirúrgico, según el caso, con el objetivo de detener la hemorragia. Si no cede a tratamiento médico sería indicación de: legrado uterino completo diagnóstico o terapéutico, histeroscopia quirúrgica, e incluso en casos de hemorragia intensa, embolización de arterias uterinas.

\section{Prurito vulvar}

Aunque no se debería considerar en la mayoría de los casos un motivo de consulta urgente, en la práctica muchas pacientes recurren a urgencias por el gran disconfort que ocasiona.

Origen: vaginitis atrófica, vulvovaginitis irritativa, vulvovaginitis infecciosa, distrofia vulvar (liquen escleroso y liquen simple crónico o hiperplasia de células escamosas) y carcinoma.

El liquen escleroso es el más frecuente. Es más frecuente en mujeres posmenopáusicas y se considera benigno, pero un $4-6 \%$ se asocia a cáncer vulvar, más frecuente en los estadios avanzados, por lo que siempre se deben remitir a consulta para su diagnóstico anatomopatológico por biopsia y tratamiento oportuno.

\section{Masa pélvica}

Una de las patologías por las que se acude a urgencias con mayor frecuencia es por la presencia de dolor abdominal, siendo en numerosas ocasiones la causa del dolor la existencia de una masa pélvica. En otros pacientes aparece como hallazgo casual a lo largo de la exploración.

Opciones para el diagnóstico etiológico de las masas anexiales:

- Ecografía, que es la mejor herramienta en la sala de urgencias para la valoración de la patología anexial ${ }^{7}$. Recomiendan la vía vaginal, pero complementada con la ecografía abdominal, 
ya que permite una imagen más amplia de la pelvis.

- TAC, indicado como una exploración complementaria a la ecografía, de utilidad en ciertos casos cuando la imagen ecográfica no sea resolutiva y existan dudas.

- RNM, ofrece una resolución del tejido excelente, pero la gran mayoría de sus indicaciones en ginecología no son urgentes.

- Laboratorio, son de menos ayuda en estas paciente, aunque en casos de dolor severo será útil un hemograma completo ya que algunas urgencias, como la torsión ovárica o el absceso tuboovárico, presentan leucocitosis.
En el caso de las masas tumorales resultan de utilidad los marcadores tumorales, fundamentalmente el CA 125.

Detectada una masa anexial, se plantearán dos cuestiones fundamentales en la asistencia de urgencias:

1. Determinar si nos encontramos ante una urgencia verdadera (como torsión de ovario, embarazo ectópico, apendicitis) o nos encontramos ante un proceso que no requiere cirugía (quiste funcional).

2. Clasificar la masa anexial como de bajo o alto riesgo de malignidad. La imagen ecográfica suele ofrecernos ya muchos signos de sospecha (Fig. 1).

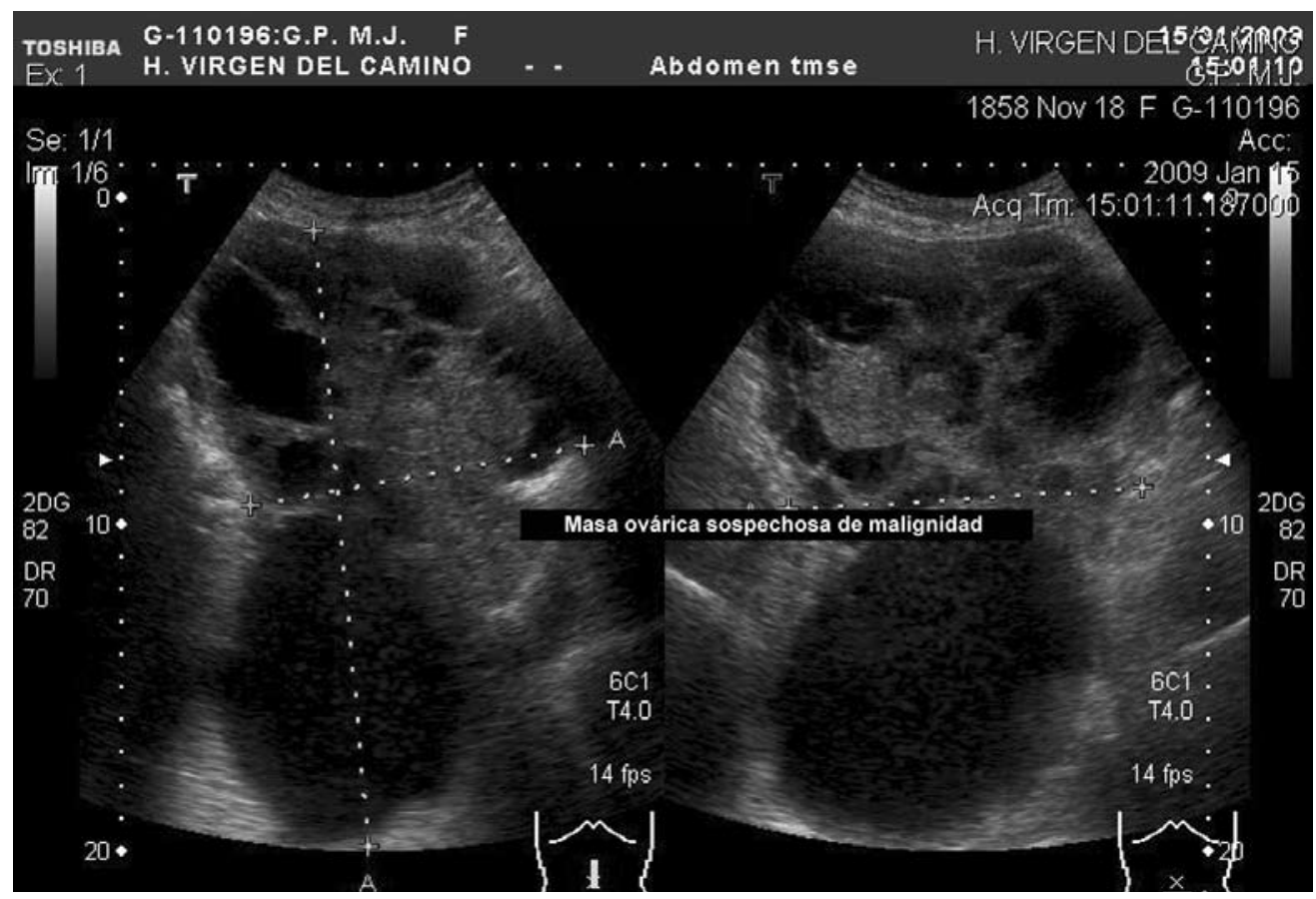

Figura 1. Masa ovárica compleja.

\section{COMPLICACIONES DE LA ENFERMEDAD Y DEL TRATAMIENTO}

\section{Obstrucción ureteral}

La obstrucción de la vía urinaria puede producirse en el contexto de un atrapamiento por neoplasia pélvica, o bien por compresión extrínseca de una masa tumo- ral, o estenosis secundarias a cirugía o radioterapia.

Existen diversos tipos de afectación ${ }^{8}$ :

- Obstrucciones unilaterales, que habitualmente cursan de manera asintomática y se descubren accidentalmente al detectar un riñón hidronefrótico en fases iniciales. 
- Obstrucciones bilaterales parciales, que pueden desencadenar cuadros de insuficiencia renal crónica, alteraciones tubulares renales con déficit en la concentración de sodio y agua (diabetes insípida nefrogénica), alteraciones de las nefronas distales y túbulos colectores con acidosis hiperclorémica e hiperpotasémica, y complicaciones como hipertensión arterial e infecciones urinarias.

- Obstrucción bilateral completa, que cursa con fracaso renal agudo con anuria.

Dentro del estudio diagnóstico podemos señalar:

- Determinación de hemograma y bioquímica (urea, creatinina, sodio, potasio, cloro, bicarbonato, calcio, albúmina); sedimento y cultivo de orina; ecografía abdominal; TAC abdominopélvico, que ayudará en la identificación de la causa de la obstrucción (Fig. 2). Las urografías o pielografías deben evitarse en los cuadros agudos. Están reservadas para casos de etiología no aclarada y de evolución lenta.

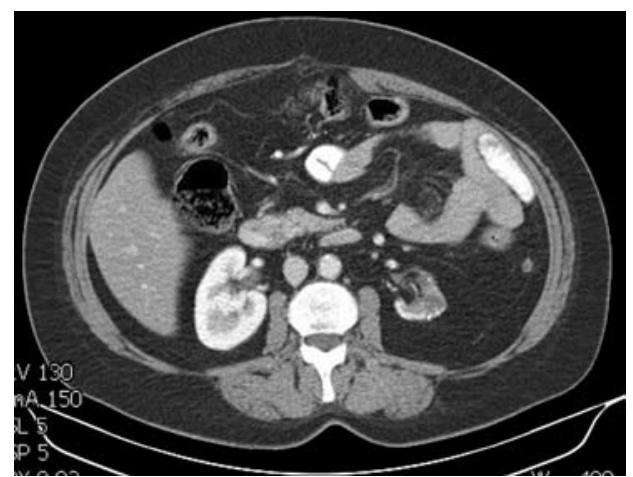

Figura 2. Necrosis renal cortical por obstrucción ureteral izquierda.

Actitud terapéutica:

- Resolución del cuadro obstructivo mediante nefrostomía percutánea o cateterización ureteral.

- Corrección de las complicaciones hidroelectrolíticas.

- Tratamiento antibiótico si existe infección asociada.

- Tratamiento quirúrgico siempre que sea posible, para extirpar la masa tumoral que engloba o comprime la vía urinaria.

\section{Carcinomatosis peritoneal}

Puede presentarse en pacientes que acuden a urgencias con síntomas de enfermedad neoplásica avanzada, como la presencia de dolor y aumento del perímetro abdominal con presencia de ascitis, náuseas y vómitos. En situaciones más extremas la paciente puede presentar síntomas de obstrucción intestinal o disnea por la presencia de derrame pleural.

En estas mujeres se plantea el manejo de su dolor (asociado muchas veces a la distensión abdominal secundaria a la ascitis), así como el diagnóstico exacto de su proceso, en aras a establecer el abordaje terapéutico preciso.

Dentro del estudio diagnóstico podemos señalar:

- La ecografía, tanto transvaginal como la abdominal, que a través de unas imágenes de sospecha de malignidad permitirá realizar un diagnóstico de sospecha de forma rápida y fiable. Valoración doppler.

- TAC para establecer si existe afectación extrapélvica y grado de afectación de la misma.

- Estudio citológico del líquido ascítico recogido tras paracentesis (utilizada como técnica terapéutica para el alivio del dolor que la ascitis ocasiona por distensión).

Para establecer el tratamiento, previamente debemos conocer el origen neoplásico de la carcinomatosis (en ocasiones el tumor primario corresponde a neoplasias de origen digestivo con metástasis en ovarios), porque las opciones terapéuticas son distintas. El estudio citológico de la ascitis puede reflejar un adenocarcinoma sin establecer origen digestivo o ginecológico) y esta dilación en el tiempo para no llegar a diagnósticos concluyentes nos anima a propugnar la realización de una laparoscopia exploradora:

- Permite biopsiar directamente las lesiones sospechosas, con lo que obtendremos un diagnóstico de certeza.

- Permite la aspiración de ascitis en el caso de que fuera preciso.

- En el caso de que sea de origen ovárico, posibilita valorar si la paciente se va a programar para cirugía citorreductora o bien remitir para protocolo de quimioterapia neoadyuvante. 


\section{Ascitis}

La distensión abdominal producida por la ascitis produce angustia, disnea, anorexia, náuseas, estreñimiento y dolor más o menos acusado.

La paracentesis es muy limitada, pero con un efecto favorecedor inmediato cuando la quimioterapia adyuvante o paliativa ha sido abandonada, o siempre que la paciente lo necesite. Cuidado no puncionar un asa, sobre todo el intestino grueso. Mejor, realizar siempre la punción guiada por ecografía. Los diuréticos, espironolactona a dosis $50-100 \mathrm{mg} / \mathrm{d}$, pueden ser eficaces en algunos casos, al menos inicialmente. También la administración de corticoides a bajas dosis disminuye el volumen de formación de ascitis. La administración de quimioterapia con fines paliativos reduce la ascitis temporalmente en un 15-20\% de los $\operatorname{casos}^{9}$.

\section{Enfermedad tromboembólica}

Implica una morbimortalidad significativa; hasta el $20 \%$ de las muertes postoperatorias son debidas a embolismo pulmonar y posee un alto riesgo de recurrencia incluso con tratamiento preventivo anticoagulante.

Las pacientes oncológicas se encuentran en un estado de hipercoagulabilidad

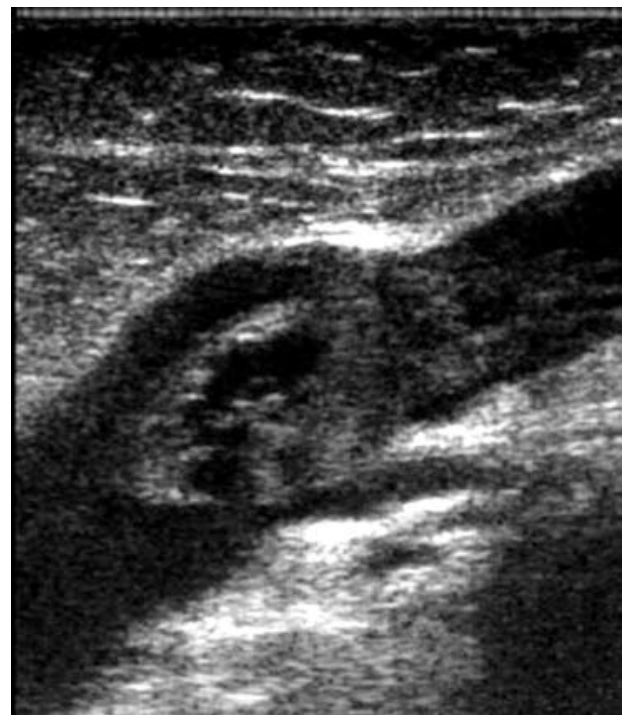

Figura 3. Imagen ecográfica de trombosis venosa. por factores de riesgo debidos al paciente, al tumor (la presencia de metástasis es un potente factor predictivo) y a los tratamientos (cirugía, quimioterapia, hormonoterapia, radioterapia, catéteres...).

\section{Trombosis venosa profunda}

La incidencia es mayor durante el primer año de seguimiento, sobre todo en los tres primeros meses al diagnóstico, y disminuye posteriormente. Afecta a venas de las piernas y pelvis con mayor frecuencia pero debido al uso de catéteres venosos centrales, está aumentando la incidencia en venas de extremidades superiores.

Los signos y síntomas no son sensibles ni específicos: menos de la mitad presentan síntomas y sólo el $40 \%$ con sospecha clínica ven confirmado el diagnóstico.

Síntomas clínicos habituales son: edema, tumefacción, dolor, calor, palpación cordón venoso y signo de Hommans positivo. No siempre están presentes en su totalidad y debe sospecharse en pacientes con cáncer que presenten hinchazón aguda de un miembro.

El diagnóstico suele confirmarse con Eco-doppler de extremidad afecta (Fig. 3), y en escasas ocasiones hace falta flebografía, prueba "gold standard".

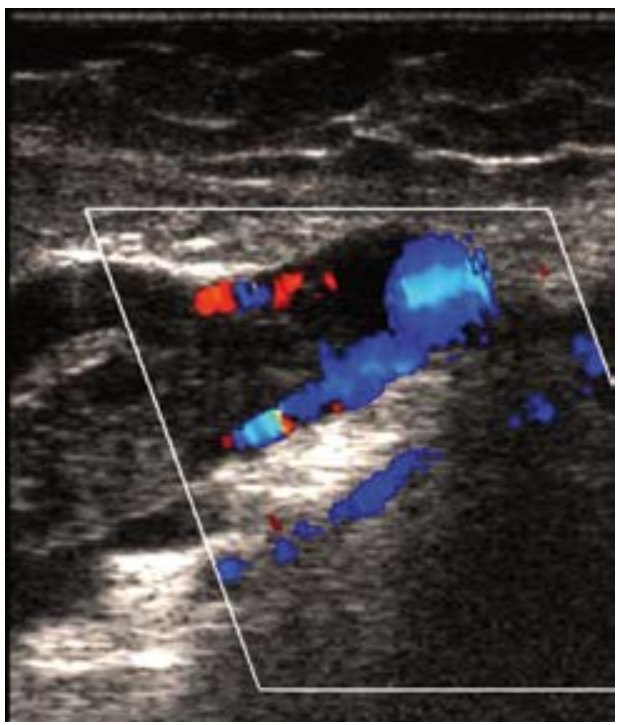


El tratamiento es prevenir el aumento del trombo y la embolización del mismo. Administrar heparina de bajo peso molecular en fase aguda, cada $24 \mathrm{~h}$ vía subcutánea, y preventivo. También heparina en infusión continua después de una dosis de carga i.v. monitorizando el tiempo de cefalina durante 5 a 7 días, y se sustituye por anticoagulantes orales, que se controla con la monitorización del tiempo de protrombina; mantener entre 3 y 6 meses si es que no hay otros factores, diferentes a la cirugía, que aconsejen mantenerlos más tiempo ${ }^{10}$.

En situación de contraindicación absoluta para la anticoagulación o episodios recurrentes a pesar de tratamiento anticoagulante adecuado, hay que plantearse la colocación de un filtro en vena cava inferior que evite embolismos.

\section{Embolismo pulmonar}

Suele ocasionarse a partir de trombos en el sistema venoso profundo de la pierna y, menos frecuentemente de venas pélvicas, renales, extremidades superiores o cavidades cardiacas derechas.

Los síntomas no son sensibles ni específicos y se requiere un alto grado de sospecha para alcanzar el diagnóstico (menos $30 \%$ presentan signos de trombosis venosa en miembros inferiores).

Presenta disnea de comienzo brusco, dolor pleural, puede haber tos, hemoptisis, fiebre y shock cardiovascular. A la exploración puede existir taquipnea, estertores, cuarto ruido, aumento del segundo tono cardiaco.

\section{Pruebas diagnósticas ${ }^{11}$}

1. Hemograma y bioquímica: no específicos. Puede existir leucocitosis, elevación GOT, LDH y VS. En 30-50\% existe elevación de troponina-I por sobrecarga cardiaca derecha y conlleva mal pronóstico.

2. Gasometría arterial: hipoxemia, hipocapnia, alcalosis respiratoria. En embolismos masivos con hipotensión y colapso respiratorio puede verse hipercapnia y acidosis metabólica.

3. Dímero-D: mayoría superior a 500 $\mathrm{ng} / \mathrm{ml}$, en pacientes neoplásicos su especificidad es insuficiente aunque sí es útil como valor predictivo negativo.

4. ECG: mayoría alterados aunque los hallazgos son inespecíficos, arritmia auricular, bloqueo rama derecha, alteración repolarización y onda $\mathrm{Q}$ en cara inferior.

5. Rx tórax: pocos cambios pero es necesario para descartar otros procesos. Pueden verse atelectasias, alteraciones parenquimatosas y derrame pleural.

6. Diagnóstico definitivo con gammagrafía de ventilación perfusión o con angiograma pulmonar con TAC.

\section{Tratamiento $^{11}$}

La mayoría de las muertes se producen en las primeras horas. Por lo tanto, ante la sospecha de embolismo pulmonar, el tratamiento es urgente y debe comenzar aún antes de confirmarlo.

1. Medidas generales: oxígeno, reposo, cabecera incorporada, tratamiento del shock. Ingreso en UCI en tromboembolismo pulmonar importante e incluso pueden necesitar maniobras de resucitación, fármacos inotrópicos y ventilación mecánica.

2. Anticoagulación: heparina de bajo peso molecular ajustada al peso y función renal.

3. Trombolisis: indicada en embolismos masivos con inestabilidad hemodinámica. En resto de los casos su uso es controvertido.

\section{Prevención de las complicaciones tromboembólicas}

En pacientes oncológicos ambulatorios la trombosis venosa profunda (TVP) es un factor de riesgo que reduce supervivencia y puede comprometer el tratamiento y respuesta al mismo en estos pacientes. No hay suficiente información disponible por el momento para recomendar o no el uso de anticoagulantes para prevenir la TVP.

La guía 2.007 ASCO recomienda la prevención en pacientes oncológicos hospitalizados por ser pacientes de alto riesgo, 
ello si no hay un sangrado activo y no hay otro tipo de contraindicación (cirugía reciente, presencia de diátesis hemorrágica, plaquetopenia $<50.000$, alteraciones de la coagulación).

La deambulación precoz junto a la compresión neumática intermitente no es suficiente para la profilaxis.

1. Buena hidratación, colocación adecuada de la paciente en mesa de operaciones evitando presiones excesivas.

2. Profilaxis con heparina subcutánea, a dosis $5.000 \mathrm{UI} / 24 \mathrm{~h}$, e iniciándose entre $30 \mathrm{~min}$ y $2 \mathrm{~h}$ antes de la cirugía. Recientemente se ha demostrado que la heparina de bajo peso molecular, enoxaparin $40 \mathrm{mg} / \mathrm{d}$ o dalteparin $5.000 \mathrm{UI} / 24 \mathrm{~h}$, es tan efectiva como la heparina fraccionada $5.000 \mathrm{UI} / 8 \mathrm{~h}$ en la prevención de trombosis con vida media más larga y menor riesgo de complicaciones; es pues el fármaco de elección ${ }^{12,13}$.

La guía 2.007 ASCO sugiere el tratamiento posthospitalario con heparina de bajo peso molecular durante 10 días e incluso 4 semanas posteriores a la cirugía, e incluso más de las 4 semanas en pacientes de alto riesgo por enfermedad residual tras cirugía, obesidad, historia previa TVP.

\section{Hemorragia}

Es frecuente y requiere atención inmediata y eficaz. Casi siempre nos encontramos a la exploración una lesión, habitualmente exofítica, o masa en vulva, vagina o cuello uterino, aunque puede ser vista inicialmente con una hemorragia masiva responsable de hipovolemia e incluso, hipotensión, requiriendo reposición intravascular inmediata.

\section{Tratamiento}

1. Coagulación de los puntos de hemorragia con agentes esclerosantes (sulfato férrico).

2. Taponamiento directo con gasa; se deja de 24 a 48 h. Puede impregnarse con solución de Monssell (sulfato férrico) que es altamente hemostático.
3. Si fracasa, se puede intentar la administración de una dosis hemostática de radioterapia en varias fracciones, que suele ser exitosa con apenas efectos secundarios.

Cuando el tratamiento conservador no tiene éxito, se requiere un control más agresivo de los vasos arteriales sangrantes. Esta situación es más frecuente en pacientes sometidas a cirugía previa y/o radiación y menos frecuentes en pacientes vistas por primera vez con la enfermedad. Habrá que valorar la opción de ligar la arteria hipogástrica o embolizar el vaso sangrante.

\section{Manejo del dolor oncológico}

Dentro del manejo del dolor en el paciente oncológico se deben mantener una serie de criterios:

- Utilizar los analgésicos de forma regular y no "a demanda", y prescribirse a intervalos fijos, según la duración de acción.

- La vía oral se considera de elección; las parenterales no representan ninguna ventaja salvo que no sea posible la vía oral o exista mal control por dicha vía.

- Sigue siendo válida la escalera terapéutica propuesta por la OMS, en la que intervienen 4 grupos de medicamentos: analgésicos no opioides, opioides menores, opioides mayores y adyuvantes (Tabla 1).

- Lo que determina el paso de un escalón a otro es la ineficacia del escalón anterior para controlar el dolor a dosis máximas.

- La utilización simultánea de dos medicamentos del mismo escalón no reporta ningún beneficio, salvo excepciones:

- codeína como rescate en tratamientos con dihidrocodeína de acción sostenida.

- morfina de liberación inmediata como rescate en tratamientos con parches de fentanilo transdérmico.

- En todos los pacientes se deben prescribir dosis de rescate. La dosis de rescate será un $10-15 \%$ de la dosis diaria pautada y puede repetirse cada hora; ahora bien, si se utilizan más de tres dosis de rescate en 24 horas será necesario reevaluar la dosis pautada. 
- Se evitará la polifarmacia, ya que la incidencia de efectos secundarios aumenta exponencialmente al aumentar el número de medicamentos.
- Al iniciar un tratamiento, se empezará con el escalón 1 ó 2 para dolores leves o moderados y con el escalón 3 para dolores severos (Tabla 2).

Tabla 1. Escala analgésica (OMS).

Analgésicos del primer escalón

\begin{tabular}{|l|c|c|}
\hline \multicolumn{1}{|c|}{ Medicamento } & Dosis & Dosis max/día \\
\hline Ac. Acetil salicílico & $500-1000 \mathrm{mg} \mathrm{c} / 4-6 \mathrm{~h}$ & $4000-6000 \mathrm{mg}$ \\
\hline Paracetamol & $500-1000 \mathrm{mg} \mathrm{c} / 4-6 \mathrm{~h}$ & $4000-6000 \mathrm{mg}$ \\
\hline Naproxeno & $500 \mathrm{mg} \mathrm{c} / 8-12 \mathrm{~h}$ & $1500 \mathrm{mg}$ \\
\hline Metamizol (Dipirona) & $575-2000 \mathrm{mg} \mathrm{c} / 6-8 \mathrm{~h}$ & $8000 \mathrm{mg}$ \\
\hline Diclofenaco & $50 \mathrm{mg} \mathrm{c} / 8 \mathrm{~h}$ & $150 \mathrm{mg}$ \\
\hline
\end{tabular}

Analgésicos del $2^{\circ}$ escalón

\begin{tabular}{|l|c|c|}
\hline \multicolumn{1}{|c|}{ Medicamento } & Dosis & Dosis max/día \\
\hline Codeína & $30-60 \mathrm{mg} \mathrm{c} / 4-6 \mathrm{~h}$ & $360 \mathrm{mg}$ \\
\hline Dihidrocodeína & $60-180 \mathrm{mg} \mathrm{c} / 12 \mathrm{~h}$ & $360 \mathrm{mg}$ \\
\hline Tramadol gotas & $50-100 \mathrm{mg} \mathrm{c} / 6-8 \mathrm{~h}$ & $400 \mathrm{mg}$ \\
\hline Codeína + paracetamol & $1-2 \mathrm{com} \mathrm{c} / 4-6 \mathrm{~h}$ & $8 \mathrm{comp}$ \\
\hline
\end{tabular}

Analgésicos del 3er escalón

\begin{tabular}{|c|l|l|l|}
\hline Medicamento & \multicolumn{1}{|c|}{ Presentaciones } & Dosis & Dosis \\
\hline Morfina & $\begin{array}{l}\text { Liberación inmediata } \\
\text { (comp. } 10 \mathrm{y} 20 \mathrm{mg}) \\
\text { Liberación sostenida } \\
(\text { comp.o caps. } 10,30,60,100 \mathrm{y} 200 \mathrm{mg})^{\mathrm{b}} \\
\text { Liberación controlada en } 24 \mathrm{~h} \\
(\text { comp. de } 30,60,120 \mathrm{y} 200 \mathrm{mg})^{\mathrm{b})}\end{array}$ & A partir de $10 \mathrm{mg} / 4 \mathrm{~h}^{\mathrm{a}}$ & No hay \\
\hline Metadona & Comprimidos $5 \mathrm{mg}$ & A partir de $60 \mathrm{mg} / 24 \mathrm{~h}$ & $\mathrm{~h}^{\mathrm{c}}$ \\
\hline
\end{tabular}

a) En pacientes debilitados puede iniciarse con $5 \mathrm{mg} / 4 \mathrm{~h}$.

b) No se pueden triturar ni fraccionar

c) En pacientes debilitados o cuando se ha saltado el $2^{\circ}$ escalón puede iniciarse con dosis menores (p.e. 10 $\mathrm{mg} / 12 \mathrm{~h}$.)

d) Su interés es limitado. En la práctica queda reducido a pacientes con intolerancia a la morfina o los que requieren rotación de opioides.

\section{Obstrucción intestinal}

La obstrucción intestinal es una de las complicaciones más frecuentes (puede alcanzar el $42 \%$ ) y preocupantes en las pacientes con cáncer de ovario, sobre todo en estadios avanzados.

Es importante diferenciar si la obstrucción intestinal en una paciente oncológica es debida a su proceso neoplásico, a adhe- rencias o a otras causas. Incluso en pacientes obstruidas en estado de recurrencia de su enfermedad, un tercio de los casos son debidos a procesos adherenciales benignos. En pacientes operadas por procesos malignos intraabdominales, las obstrucciones debidas a recurrencia del cáncer aparecen más tempranamente tras la cirugía que las secundarias a adherencias (21 frente a 61 meses). 
Tabla 2. Algoritmo del manejo del dolor oncológico.

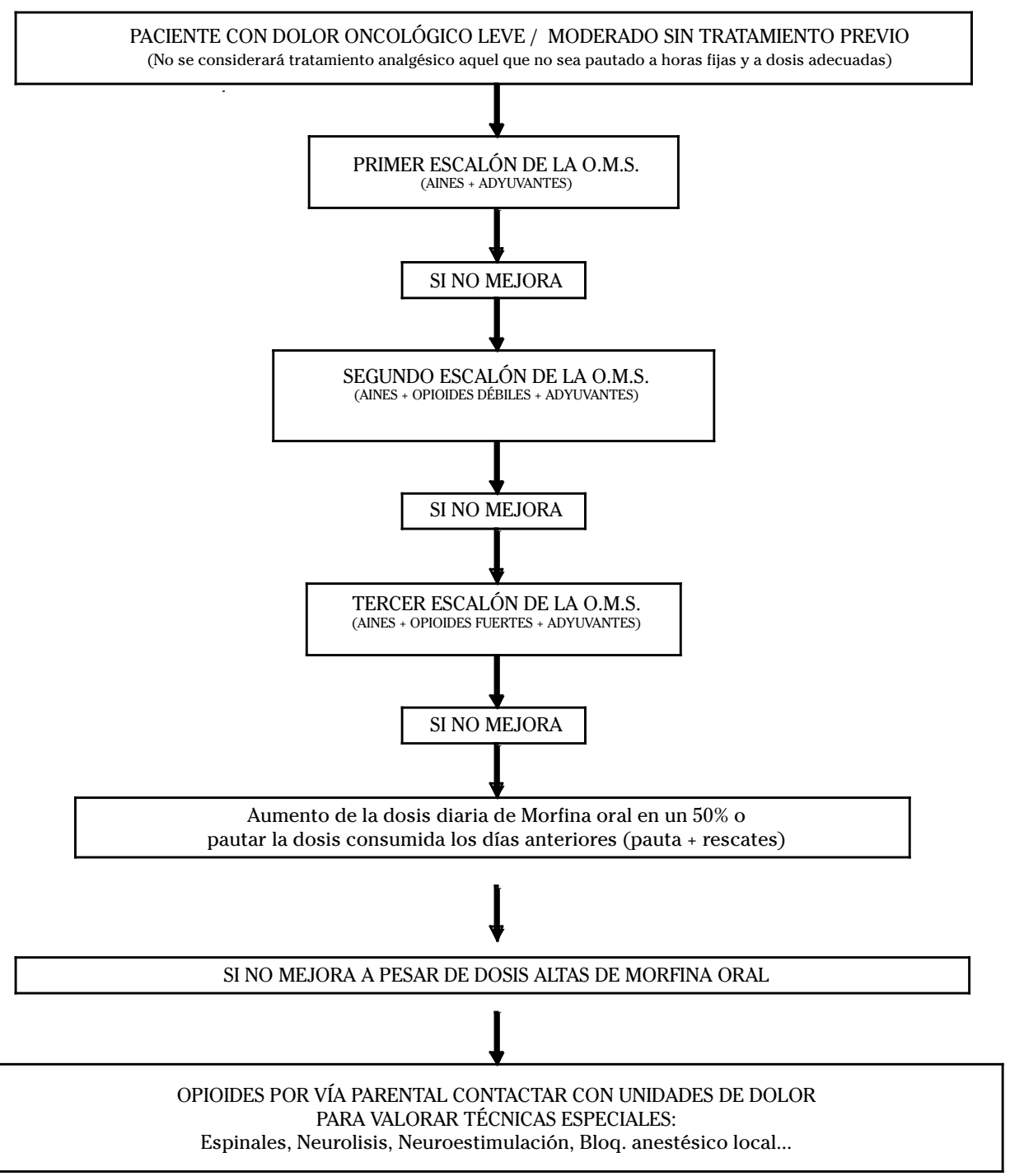

Aunque no existe unanimidad en la literatura, se pueden establecer una serie de recomendaciones:

- El planteamiento quirúrgico puede establecerse en pacientes en las que no se sospecha recurrencia del cáncer y pre- senta un largo intervalo de tiempo entre el diagnóstico de su proceso neoplásico y el desarrollo del cuadro obstructivo.

- En las pacientes con obstrucción parcial, recurrencia tumoral, corto intervalo de tiempo desde la cirugía o exis- 
te sospecha de isquemia intestinal, se mantendrá el tratamiento médico conservador. En estos casos el TAC puede ayudar a diagnosticar la causa de la obstrucción.

Cuando una obstrucción es secundaria a su proceso neoplásico y ni el tratamiento médico conservador ni la cirugía pueden aliviarle, precisará sonda nasogástrica continua y administración de alimentación parenteral. En estos casos habrá que valorar si es subsidiario de tratamiento quimioterápico que pueda mejorar su cuadro obstructivo o bien ser derivada para cuidados sintomáticos paliativos. En algunos estudios se ha comprobado la utilidad de algunos análogos de la somatostatina en el alivio de los vómitos en pacientes terminales con obstrucción intestinal cuando los antieméticos tradicionales fallan ${ }^{14}$.

Otra de las opciones paliativas, según la localización, consiste en la colocación de endoprótesis a nivel de colon.

\section{Linfocele abdominal}

Es una complicación rara (3\% de las operadas) que se origina a las 2 semanas de la cirugía. Lo habitual es que cursen asintomáticas y se detecten en estudios radiológicos de control. En otras ocasiones puede originar clínica de dolor por distensión y compresión de estructuras vecinas, pudiendo desencadenar, según localización, cuadro de hidronefrosis.

Prevención: dejar abierto el peritoneo, de manera que evitemos su encapsulación.

Dentro de las opciones terapéuticas están la aspiración simple mediante la colocación de un drenaje externo guiado por ecografía o TAC, con una tasa elevada de recurrencias que puede llegar al 50\%; la inyección de sustancias esclerosantes tipo povidona yodada o alcohol, con un alto riesgo de sobreinfección; y por último, el drenaje quirúrgico mediante la creación de una ventana peritoneal, vía abierta o laparoscópica, con extirpación de parte del peritoneo que cubre el linfocele para evitar su recidiva.

\section{Linfocele inguinal y linfedema de extremidades inferiores}

Tras la realización de una linfadenectomía inguinal, las complicaciones más habi- tuales son la infección y dehiscencia de la herida, así como el linfedema de extremidades inferiores, alcanzando una incidencia del $60 \%$. El riesgo aumenta con la extensión de la linfadenectomía, ganglios infiltrados, aplicación de radioterapia adyuvante en la zona, la extirpación del cayado de la safena $\mathrm{y}$ en pacientes mayores de 65 años $^{15}$.

Para la prevención de dichas complicaciones, las medidas se basan en el mantenimiento del drenaje mientras persista salida de contenido linfático, en el vendaje compresivo, mantenimiento en alto de la extremidad y ejercicios rehabilitadores que favorezcan el drenaje linfático.

En aquellos casos que la paciente acude urgente por linfocele inguinal, la pauta de actuación consistirá en el drenaje de la colección bajo cobertura antibiótica para la prevención de infección de la herida, con posterior vendaje compresivo inguinal.

\section{Enteritis post-radioterápica}

Es preciso diferenciar entre la enteritis aguda, que se produce durante o poco después de finalizar el tratamiento radioterápico y se resuelve en dos a seis semanas, y la enteritis crónica, que se desarrolla habitualmente a partir de los seis meses de acabar el tratamiento.

Pueden presentar síntomas de diarrea crónica, o incluso síntomas obstructivos con o sin complicaciones como fístulas o perforaciones.

Factores de riesgo: dosis de radioterapia, técnica radioterápica inadecuada, edad avanzada, administración postquirúrgica, presencia de adherencias por cirugías previas, enteritis rádica aguda previa ${ }^{16}$.

Dentro del estudio diagnóstico, además de una analítica para valorar el estado nutricional, se debe solicitar una placa de tórax y abdomen, así como un test del aliento para descartar sobrecrecimiento bacteriano en pacientes con clínica de diarrea, dolor abdominal, náuseas y meteorismo. En los casos en los que se prevea cirugía, solicitaremos pruebas para valorar la extensión de afectación intestinal: TAC abdominal, tránsito intestinal o colonoscopia.

Protocolo terapéutico:

- Dieta baja en residuos, sin lácteos en caso de intolerancia a la lactosa y con 
glutamina añadida para la recuperación de la mucosa entérica.

- Antidiarreicos tipo loperamida (contraindicados en caso de obstrucción).

- Antibióticos si se confirma sobrecrecimiento bacteriano.

- Nutrición parenteral en casos de enteritis severa, que puede ser temporal o definitiva.

- La cirugía está indicada en el íleo persistente, tras la formación de fístulas y en casos de adherencias masivas.

\section{Proctitis post-radioterápica}

La proctitis aguda se inicia en las seis primeras semanas tras finalización de la radioterapia, provocando diarrea, urgencia, tenesmo, a veces rectorragia, y se resuelve sin tratamiento en dos a seis semanas.

En el caso de la proctitis crónica la aparición se establece en torno a los 9-14 meses de finalización del tratamiento. Los síntomas son parecidos y el diagnóstico se establece mediante toma de biopsia tras control colonoscópico, que aunque no específica, permite sobre todo descartar otras causas. Dentro del tratamiento se han utilizado reblandecedores de heces, enemas de sucralfato, electrocoagulación, dilatación endoscópica y otras medidas (sulfasalacina, aminosalicilatos, corticoides,...) con limitada experiencia.

\section{BIBLIOGRAFÍA}

1. Ardanaz E, Moreno C, Pérez de Rada ME, EzponDA C. Incidencia y mortalidad por cáncer en Navarra, 1998-2002. Evolución en los últimos 30 años. An Sist Sanit Navar 2007; 30: 245-270.

2. Colomer R, Alva E, Llombart A. Planificación asistencial de la Oncología Médica en España 2008. Primer libro blanco de la Oncología Médica en España.

3. Goldstein RB, Bree RL, Benson CB, Benacerraf BR, Bloss JD, Carlos R et al. Evaluation of the woman with postmenopausal bleeding: Society of radiologists in ultrasound-Sponsored Consensus Conference Statement. J Ultrasound Med 2001; 20: 1025-1036.

4. Shaw RW, Allen I, Harper MA, Hempsall V, Hourahane J, Rees MCP, Smith SK, Sutton P. The management of menorrhagia in secondary care.
Good Medical Practices Guidelines. Royal College of Obstetricians and Gynaecologists, London 2000.

5. Karlsson B, Grandberg S, Wikland M, Ylostalo P, Torvid K, Marsal K et al. Transvaginal ultrasonography of the endometrium in women with postmenopausal bleeding-a Nordic multicenter study. Am J Obstet Gynecol 1995; 173: 1488-1494.

6. Mossa B, Imperato F, Marziani R, Perniola F MeLluso J, Perniola G et al. Hormonal replacement therapy and evaluation of intrauterine pathology in postmenopausal women: a tenyear study. Eur J Gynaecol Oncol 2003; 24: 507-512.

7. Morgan A. Anexal mass evaluation in the emergency department. Emerg Med Clin North 2001; 19: 799-816.

8. KLAHR S. Urinary tract obstruction. En: Scherier $\mathrm{R} \mathrm{W}$ editor. Disease of the kidney and urinary tract $7^{\text {th }}$ edition. Lippincott Williams \& Wilkins Publisher, 2001.

9. LiCKISS J NoRELLE. Pallitive caren and pain management. En: Berek and Hacker, eds. Practical gynecology oncology. Baltimore 1994: 687.

10. Hutten BA, Prins MH. Duration of treatment with vitamin $\mathrm{K}$ antagonists in symptomatic venous thromboembolism. Cochrane Database of Systematic Reviews. Issue 4, 2000.

11. Khorana M, Francis CW, Culakova E, Lyman GH. Risk factors for chemotherapy-associated venous thromboembolism in a prospective observational study. Cancer 2005; 104 : 2822-2829.

12. Van Dogen, Van den Belt AGM, Prins MH, LenSING AWA. Fixed-dose subcutaneous lowmolecular-weight heparins versus adjusteddose unfractionated heparin for venous thromboembolism. Cochrane Database of Syst Rev 4, 2000.

13. PRINS MH. Vitamin K antagonists or lowmolecular-weight heparin for the long-term managemente of symptomatic venous thromboembolism. Cochrane Database of Systematic Reviews. Issue 4, 2000.

14. Matulonis UA, Seiden MV, Roche M, Krasner C, FULLER AF, ATKINSON T et al. Long-acting octreotide for the treatment and symptomatic relieve of bowel obstruction in advanced ovarian cancer. J Pain Sympton Manage 2005; 30: 563.

15. Gaarenstroom KN, Kenter GG, Trimbos JB, Agous I, A, Amant, Peters AA et al. Postoperative complications after vulvectomy and inguinofemoral lymphadenectomy using separate groin incisions. Int J Gynecol Cancer 2003; 13: 522.

16. NGUYEN NP. Current concepts in radiation enteritis and implications for future clinical trials. Cancer 2002; 95: 1151. 